\title{
Accommodation: How You See It, How You Don't
}

\author{
J. L. Platt ${ }^{a, b, *}$ \\ Departments of a Surgery and ${ }^{\mathrm{b}}$ Microbiology and \\ Immunology, University of Michigan, Ann Arbor, MI \\ *Corresponding author: Jeffrey L. Platt, plattj@umich.edu
}

\section{Received 13 May 2011, revised 26 May 2011 and accepted for publication 26 May 2011}

Accommodation refers to a condition in which a transplant sustains little or no injury and functions normally despite the presence of anti-donor antibodies in the circulation of the recipient. We first observed accommodation in the 1980's in the setting of ABO-incompatible kidney transplantation. At that time, $75 \%$ of kidneys incompatible for blood group-A or -B were rejected within 3 months. However, Guy Alexandre and a few others found that rejection could be averted and enduring function achieved if the offending antibodies were depleted at the time of surgery. Probing the surprising success we found that in some recipients the anti-blood antibodies returned to the circulation and in all cases the kidneys continued to express the corresponding antigen. We figured then that the grafts might have acquired resistance to injury (1). After observing a similar phenomenon in cardiac xenografts, we applied the term 'accommodation' to denote the possibility that the graft and/or recipient had changed in such a way that what was once lethal was no longer so (2). Notwithstanding occasional reports on highly sensitized recipients (3), accommodation has been seen rarely in recipients presensitized to HLA (perhaps because anti-HLA antibodies include high affinity lgG, presumably reflecting somatic hypermutation characteristic of $T$ cell-dependent and not $T$ cell-independent responses that attack blood group and xenogeneic antigens) and de novo produced anti-HLA antibodies presage bad outcomes (4). Whether accommodation can be achieved reliably in presensitzed recipients, how it occurs and why it is not seen more often should compel interest.

In this issue of AJT, Chen et al. (5) report that monkeys previously sensitized by skin transplantation and treated with cobra venom factor (CVF) and with conventional immunosuppression agents can accept kidney grafts. The monkeys had anti-donor antibodies both before and after transplantation and the grafts had normal histology indicating the establishment (or development if you wish) of accommo- dation. Clearly then, accommodation can be achieved in presensitized primates and that answer should excite clinicians and scientists.

Although Chen and co-workers did not attempt to test how accommodation occurs, their discussion nicely frames this question by commenting that CVF might "bring about" accommodation or "allow it to assert itself" by preventing destruction of the graft. The latter explanation seems most appealing to the authors as they call the CVF used "an extremely potent anti-complement protein". This CVF might allow anti-MHC antibodies to induce protective changes, as described independently from the laboratories of Dalmasso (6), Delikouras (7) and Jindra (8), rather than injury. On the other hand, the CVF used might actually induce accommodation, not by inhibiting complement but rather by activating it. The CVF from Naja kaouthia (Naja kaouthia CVF) activates both C3 and C5, generating C3a and C5a and it generates small amounts of terminal complexes that can insert in cell membranes, causing 'reactive lysis' of susceptible cells, like heterologous erythrocytes, and possibly protection of less susceptible cells, such as endothelial cells. The answer to whether it is anti-MHC antibodies and/or activated complement proteins (or something else) that protects grafts from lethal injury could eventuate in new therapeutics in many fields.

If anti-MHC antibodies, which some think are made by most graft recipients, can induce both rejection and accommodation, why is accommodation not seen more often? Chen et al. (5) seem to think the amount of anti-MHC antibody produced explains the outcome-large amounts cause rejection, small amounts accommodation. Consistent with this view they note that monkeys treated with CVF had lower levels of anti-donor antibodies than monkeys not given CVF and take from this result that "... CVF significantly attenuated [the] induced antibody response...". However, we and others before us have observed that perfused organs and organ grafts can absorb huge amounts of anti-donor antibodies from blood and in some cases anti-donor antibodies may not be seen at all until a graft is rejected or removed (9). Thus, if accommodation allows a graft to continue being perfused it will enable removal of much or all of the anti-MHC antibody produced, while rejection, whether triggered or not by antibodies, will prevent absorption by decreasing the rate blood flows through the graft. One implication of the second potential mechanism is that assays for anti-donor antibodies may well underestimate or entirely miss humoral responses to a graft donor and hence accommodation. If that is so then 
we will not see accommodation in many cases when it is present and our original definition of accommodation, the simultaneous presence of anti-donor antibodies and an unblemished graft, should be abandoned. Nor do the results of Chen et al. encourage detection of "protective genes or proteins" as a marker of accommodation because they show (but do not discuss), that these genes and proteins are also seen in rejection. To understand better what accommodation is and how prevalent it may be, we need a better way of seeing it.

\section{Acknowledgment}

Supported by grants from the NIH (HL52297 and HL79067).

\section{Disclosure}

The author of this manuscript has no conflict of interest to disclose as described by the American Journal of Transplantation.

\section{References}

1. Bannett AD, McAlack RF, Morris M, Chopek M, Platt JL. ABO incompatible renal transplantation: $A$ qualitative analysis of native endothelial tissue $\mathrm{ABO}$ antigens after transplant. Transplant Proc 1989;21:783-785

2. Platt JL, Vercellotti GM, Dalmasso AP, et al. Transplantation of discordant xenografts: A review of progress. Immunol Today 1990;11:450-456.

3. Salama AD, Delikouras A, Pusey CD, et al. Transplant accommodation in highly sensitized patients: A potential role for $\mathrm{Bcl}-\mathrm{xL}$ and alloantibody. Am J Transplant 2001;1:260-269.

4. Ho EK, Vlad G, Vasilescu ER, et al. Pre- and posttransplantation allosensitization in heart allograft recipients: Major impact of de novo alloantibody production on allograft survival. Hum Immunol 2011;72:5-10.

5. Chen S, Zhong S, Xiang Y, et al. Complement inhibition enables renal allograft accommodation and long-term engraftment in presensitized nonhuman primates. Am J Transplant 2011;11:2057-2066.

6. Dalmasso AP, Benson BA, Johnson JS, Lancto C, Abrahamsen MS. Resistance against the membrane attack complex of complement induced in porcine endothelial cells with a Gal alpha(13)Gal binding lectin: Up-regulation of CD59 expression. J Immunol 2000;164:3764-3773.

7. Delikouras A, Fairbanks LD, Simmonds AH, Lechler RI, Dorling A. Endothelial cell cytoprotection induced in vitro by allo- or xenoreactive antibodies is mediated by signaling through adenosine A2 receptors. Eur J Immunol 2003;33:3127-3135.

8. Jindra PT, Hsueh A, Hong L, et al. Anti-MHC class I antibody activation of proliferation and survival signaling in murine cardiac allografts. J Immunol 2008;180:2214-2224.

9. Platt JL. Antibodies in transplantation. Discovery Medicine 2010;10(51):125-133. 